\title{
Proposição de uma sistemática para a avaliação da biocompatibilidade dos produtos de uma empresa de artigos médicos e hospitalares de Pelotas (RS)
}

\section{Proposition of a systematic for the biocompatibility evaluation of the products of a medical company in Pelotas (RS)}

Ariane Ferreira Porto Rosa ${ }^{1 *}$, Geórgia Souto $^{1}$, Rogério Royer ${ }^{1}$, Renata Heidtmann Bemvenuti ${ }^{1}$

\section{RESUMO}

A biocompatibilidade é definida como a comprovação da relação segura entre produtos médicos e seus usuários. 0 presente trabalho deste artigo foi desenvolvido utilizando como base as regulamentações e normas da Agência Nacional de Vigilância Sanitária e da Associação Brasileira de Normas Técnicas, ISO 10993-1 (Avaliação e ensaio dentro de um processo de gerenciamento de risco) e ISO 14971 (Produtos para a saúde: aplicação de gerenciamento de risco a produtos para a saúde). Foi apresentada uma proposta de metodologia geral para a avaliação da biocompatibilidade de produtos. Além disso, foi desenvolvido um estudo de caso em uma empresa do ramo médicohospitalar. Este trabalho segmentou-se em cinco etapas, sendo elas: revisão da literatura, estudo das normas técnicas, estudo de caso, proposição da sistemática no estudo de caso e conclusão. 0 estudo de caso foi dividido em: (i) mapeamento do processo produtivo da empresa estudada; (ii) análise de dados existentes; (iii) gerenciamento de risco do processo da empresa; (iv) proposição da sistemática para avaliação biológica. A pesquisa desenvolvida neste trabalho cumpriu com seus objetivos iniciais, auxiliando a empresa estudada a realizar sua própria avaliação de biocompatibilidade e gerando um método genérico e replicável.

Palavras-chave: Biocompatibilidade; Avaliação Biológica; Agência Nacional de Vigilância Sanitária.

\begin{abstract}
Biocompatibility is defined as proof of the safe relationship between medical products and their users. The present work of this article was developed based on the regulations and standards of the National Health Surveillance Agency and the Brazilian Association of Technical Standards, ISO 10993-1 (Assessment and testing within a risk management process) and ISO 14971 (Products for health: applying risk management to health products). A proposal for a general methodology was presented for the evaluation of the biocompatibility of products. In addition, a case study was developed in a company in the medical-hospital sector. This work was divided into five stages, namely: literature review, study of technical standards, case study, proposition of the systematic in the case study and conclusion. The case study was divided into: (i) mapping the productive process of the studied company; (ii) analysis of existing data; (iii) risk management of the company's process; (iv) proposition of the systematic for biological evaluation. The research developed in this work fulfilled its initial objectives, helping the studied company to carry out its own assessment of biocompatibility and generating a generic and replicable method.
\end{abstract}

${ }^{1}$ UFPel - Universidade Federal de Pelotas. *E-mail: afprosa61@ gmail.com 
Keywords: Biocompatibility; Biological Evaluation; National Health Surveillance Agency.

\section{INTRODUÇÃO}

O aumento da concorrência no mercado global de produtos e serviços faz com que as empresas busquem a constante inovação no desenvolvimento de novos produtos. Segundo Rozenfeld et al. (2006), o Processo de Desenvolvimento de Produto (PDP) é considerado cada vez mais um processo decisivo para a competitividade das empresas, considerando a gradativa internacionalização dos mercados.

No segmento de produtos para a saúde, o processo de desenvolvimento de produto é extremamente crítico devido ao propósito de uso dos produtos criados. Esses produtos devem ser arquitetados buscando o maior nível de qualidade possível, inversamente proporcional ao risco na utilização do equipamento. Devido a isso, faz-se necessária a avaliação biológica desses materiais, a fim de garantir a segurança e qualificação do artigo médico-hospitalar.

Segundo Marshall Junior et al. (2012), as legislações de defesa do consumidor, além de normas internacionais, transformaram definitivamente o propósito da qualidade dentro das organizações. Nesse cenário, a International Organization for Standardization (ISO) - em português Organização Internacional para a Padronização - é uma entidade referência que promove a normatização de empresas e produtos para manter a qualidade permanente.

Essa organização criou a Norma ISO 10993 - Avaliação biológica de produtos para a saúde, elaborada para normatizar a garantia da segurança dos biomateriais. A norma ISO 10993 Parte 1, intitulada "Avaliação e ensaio dentro de um processo de gerenciamento de risco", tem como principal objetivo a proteção dos seres humanos contra potenciais riscos biológicos decorrentes da utilização de produtos para a saúde. Ela foi compilada a partir de inúmeras normas internacionais e nacionais e guias relativos à avaliação biológica de produtos para a saúde e tem como papel servir de guia para as empresas planejarem as avaliações da biocompatibilidade dos seus produtos.

Ao mesmo tempo em que os produtos para a saúde se modernizam, também são atualizadas as normas e resoluções que regem o uso dos mesmos. Atualmente no Brasil, a avaliação de biocompatibilidade é uma exigência da Agência Nacional de Vigilância 
Sanitária (ANVISA) para os produtos para a saúde. Faz-se necessário que as empresas manufatureiras do ramo da saúde comprovem que seus artigos não virão a causar danos aos seus usuários, uma vez que os mesmos são projetados para dar aporte a pessoas com patologias. Com base nisso, este trabalho tem como finalidade mostrar o que é e como se dá a realização dessa avaliação.

Este tema tem referências escassas no Brasil. Assim, busca-se com esta pesquisa elaborar uma sistemática que sirva para orientar outras empresas que futuramente venham a analisar a biocompatibilidade de seus produtos. Este trabalho poderá contribuir com os poucos trabalhos existentes na bibliografia brasileira sobre essa temática, tendo em vista que na literatura em português sobre esse tema os trabalhos encontrados apresentam testes de biocompatibilidade, mas não aprofundam aspectos teóricos, metodológicos e de pesquisa bibliográfica, os quais fazem parte dos objetivos deste trabalho.

Este artigo encontra-se estruturado em cinco seções. A primeira seção mostra esta introdução contendo um panorama geral do trabalho realizado. A segunda seção apresenta a revisão teórica necessária para a realização do estudo. Na seção 3 descreve-se a metodologia utilizada. A seção 4 apresenta os resultados do estudo de caso realizado. $\mathrm{Na}$ seção 5 são apresentadas as conclusões deste trabalho. Por fim, são apresentadas as bibliografias referenciadas.

\section{REFERENCIAL TEÓRICO}

Nesta seção será apresentada a revisão bibliográfica acerca dos principais assuntos abordados neste trabalho: Processo de Desenvolvimento de Produtos; Produtos para saúde e Segurança Biológica.

\subsection{Processo de Desenvolvimento de Produtos}

Segundo Toledo et al. (2008) o Processo de Desenvolvimento de Produtos (PDP) é um importante processo de negócio que influencia diretamente o aumento da competitividade das empresas. Para Clark e Fugimoto (1991) e Weelwright e Clark (1992) o sucesso do PDP depende em grande parte da maneira como este processo é gerenciado. 
Na literatura, entre os principais modelos de PDP encontrados estão os modelos Stage Gates proposto por Cooper (1996), o modelo do Funil de Desenvolvimento de Wheelwright e Clark (1992) e o modelo integrado de Rozenfeld et al. (2006).

Um processo Stage Gates é um mapa conceitual e operacional para mover novos projetos de produtos da ideia até o lançamento e além, um plano para gerenciar o processo de desenvolvimento de novos produtos (PDNP) para melhorar a eficácia e a eficiência (COOPER, 2008). Nesse modelo, há a concepção da ideia e um número variável de estágios, dependendo da complexidade do produto/processo, comumente cinco estágios, até o lançamento do produto. Entre cada estágio existem os gates, que são pontos de decisão que avaliam a ideia e o desenvolvimento da mesma ao longo do projeto.

O processo do Funil de Desenvolvimento proposto por Wheelwright e Clark (1992) é iniciado com ideias de produtos e o desenvolvimento de conceitos. Nessa fase ainda há muitas incertezas quanto ao projeto, pois as possibilidades de como e com qual ideia prosseguir são inúmeras. Através do filtro 1, os produtos com maior chance de sucesso são aprovados para o portfólio. Assim começa a fase 2, onde é realizado o estudo de viabilidade e o detalhamento dos produtos. Posteriormente, as ideias passam pelo filtro 2 e caso aprovadas novamente, dá-se início à fase 3. Nessa última fase ocorrem as etapas de planejamento, execução e entrega do projeto final dos produtos a serem fabricados.

O modelo integrado de Rozenfeld et al. (2006) subdivide-se em três macrofases, que por sua vez dividem-se em nove microfases no detalhamento do projeto. Na macrofase de Pré-Desenvolvimento devem ser realizados a definição dos projetos que a empresa deseja priorizar (levando-se em consideração suas limitações como capital, tecnologia, recursos humanos), o alinhamento estratégico para analisar se o projeto poderá atingir seu objetivo e a determinação de se o produto será realmente desenvolvido. A macrofase de Desenvolvimento divide-se em: (i) Projeto Informacional - tem como finalidade definir as especificações-meta, que são os requisitos para o desenvolvimento do produto; (ii) Projeto Conceitual - objetiva desenvolver soluções para traduzir e realizar as especificações-meta definidas anteriormente; (iii) Projeto Detalhado - integra os ciclos de detalhamento, aquisição e melhoria; (iv) Preparação da Produção - consiste em desenvolver protótipos, realizar testes e produzir um lote piloto.; (v) Lançamento do Produto - lançamento do produto no mercado, junto aos serviços de manutenção e assistência técnica. A última macrofase é a de Pós-Desenvolvimento, que se resume em acompanhar o desempenho do produto no mercado e futuramente descontinuar o mesmo. 


\subsection{Produtos para a saúde}

A Agência Nacional de Vigilância Sanitária (ANVISA) não tem uma definição certa para produtos para a saúde, porém ela emprega o termo "produto médico". Esse termo foi definido na Resolução da Diretoria Colegiada (RDC) $n^{\circ}$ 2, de janeiro de 2010, que o descreve como "Equipamento, aparelho, material, artigo ou sistema de uso ou aplicação médica, odontológica ou laboratorial, destinado à prevenção, diagnóstico, tratamento, reabilitação ou anticoncepção e que não utiliza meio farmacológico, imunológico ou metabólico para realizar sua principal função em seres humanos, podendo, entretanto, ser auxiliado em suas funções por tais meios”.

O ramo de produtos médicos, também chamado de produtos para saúde, está sujeito a um rigoroso conjunto de normas, nacionais e internacionais. Isso implica que o sucesso dos processos de pesquisa e desenvolvimento do setor não prescinde da aplicação das boas práticas para desenvolvimento de produtos. Assim sendo, a gestão de projetos é particularmente relevante para o setor de produtos para saúde (OLIVEIRA et al, 2012).

Dada a complexidade dos aspectos regulatórios e processos técnico-científicos envolvidos na produção de produtos para a saúde, se faz necessário que medidas rígidas de controle sejam empregadas no processo de desenvolvimento desses produtos. Tendo isso em vista, é essencial que os produtos para a saúde sejam avaliados a fim de atestar sua segurança biológica.

\subsection{Segurança Bilológica}

Para Tinkler (2001) a segurança biológica abrange todos os aspectos da composição ou desempenho de um dispositivo médico que resultem em riscos para pacientes ou usuários, relacionados às propriedades biológicas do dispositivo ou à efeitos biológicos que ocorrem devido a exposição a ele. Isso é, por definição, o termo "segurança biológica" significa a inexistência da probabilidade de ocorrência de reações adversas em pacientes, advindas da interação com produtos para a saúde. Dentro do contexto da segurança biológica, temos de analisar uma parte crucial que é a biocompatibilidade. 
A palavra biocompatibilidade significa compatibilidade com tecidos ou órgãos vivos, por não haver toxicidade, nocividade nem provocar rejeição imunológica (FERREIRA, 2014). Ou seja, é definida como a capacidade do material analisado ter uma resposta apropriada à sua finalidade sem causar reações indesejáveis como alergias, inflamações ou toxicidade quando em contato com tecidos humanos e fluidos como o sangue.

Segundo Tinkler (2001), dizer que um produto é biocompatível não necessariamente significa que o produto não apresente algum risco, mas que o mesmo performará de forma a ter uma resposta apropriada do seu usuário. Assim, a avaliação da biocompatibilidade de um produto deve comprovar que o mesmo cumprirá as funções para as quais foi projetado.

Espera-se que produtos para a saúde não resultem em danos aos pacientes que os utilizam (TINKLER, 2001). Porém, mesmo que os fabricantes desses produtos possuam métodos de controle de qualidade, erros no processo de produção podem resultar em equipamentos sem segurança biológica. Dessa forma, estudos de biocompatibilidade se fazem necessários para garantir, desde o projeto, que o produto será confiável.

\subsection{Avaliação da Segurança Bilológica}

A avaliação da segurança biológica pode ser realizada através de pesquisas e/ou testes, todos com o mesmo fim de assegurar a garantia de funcionamento próprio dos produtos para a saúde. Para isso, a empresa deve possuir extensa documentação que detalhe sobre seus produtos e processos. Para realizar a avaliação da segurança biológica, deve-se ir ao encontro dos requisitos regulatórios nacionais, pois os mesmos mostrarão que linha de avaliação deve ser seguida e o que é necessário para realizá-la.

A ANVISA é a agência reguladora que controla os produtos e serviços vinculados à saúde, como medicamentos, cosméticos, saneantes, entre outros. Esta agência é responsável pela aprovação da comercialização desses produtos. Em nível mundial temos a International Organization for Standardization (ISO) como principal órgão de normatização utilizado para a definição de requisitos regulatórios. A ISO tem como objetivo principal aprovar normas internacionais em todos os campos técnicos, como normas técnicas, classificações de países, normas de procedimentos e processos, e etc. 
No Brasil, a ANVISA utiliza-se da Associação Brasileira de Normas Técnicas (ABNT), que rege as normas brasileiras e/ou normas internalizadas. Esse órgão faz uso das resoluções da ISO e as traduz para serem usadas em território nacional. Sendo assim, é a representante da ISO no país.

Em 2015, a ANVISA - juntamente ao Ministério da Saúde, publicou a Resolução da Diretoria Colegiada (RDC) $n^{\circ} 40$, que dispõe sobre o cadastro para controle sanitário de produtos médicos. Para adequar-se à $\mathrm{RDC} \mathrm{n}^{\circ} 40$, os produtos devem ter um dossiê técnico, que é um documento que descreve os elementos que compõem o produto, indicando as características, a finalidade, o modo de uso, o conteúdo, os cuidados especiais, os potenciais riscos, o processo produtivo e as informações adicionais.

Dentre os relatórios exigidos para compor o dossiê técnico, existe o relatório de avaliação da biocompatibilidade. Esse estudo deve determinar se o produto médico tem a capacidade de performar com uma resposta apropriada do paciente em uma aplicação específica. Duas normas são imprescindíveis para a avaliação da segurança biológica dos produtos para a saúde. São elas: (i) ISO 10993, Avaliação biológica de produtos para a saúde; (ii) ISO 14971, Produtos para a saúde: aplicação de gerenciamento de risco a produtos para a saúde.

A norma ISO 10993, Avaliação biológica de produtos para a saúde, tem como principal objetivo a proteção dos seres humanos contra potenciais riscos biológicos decorrentes da utilização de produtos para a saúde. Esta norma foi compilada a partir de inúmeras normas internacionais e nacionais e guias relativos à avaliação biológica de produtos para a saúde. Ela deve servir como guia para que as empresas planejem sua avaliação biológica. Não oferece métodos rígidos para a avaliação, porém define pontos críticos que devem ser considerados.

A avaliação biológica deve avaliar a pertinência de: (i) características físicas e químicas dos materiais; (ii) histórico de uso clínico ao longo dos anos de fabricação e comercialização do produto; (iii) dados toxicológicos do produto e de seus materiais; (iv) procedimentos para ensaios. Buscando outras alternativas, a avaliação pode incluir dados de experiência clínica e pós-clínica, pois dependendo da análise desses dados, pode ser concluído que nenhum ensaio seja necessário. 
Além disso, deve-se avaliar a significância dos seguintes aspectos: (i) materiais de fabricação; (ii) aditivos, contaminantes de processo e resíduos; (iii) substâncias lixiviáveis; (iv) produtos de degradação; (v) demais componentes; (vi) desempenho e características do produto final; (vii) características físicas do produto final. Basicamente, investiga-se todo o processo de fabricação dos produtos com suas entradas e eventuais interferentes.

Uma parte crucial da norma é classificar e separar os produtos para a saúde conforme seu contato com o corpo humano e a duração do mesmo. Após a categorização do produto, para iniciar o processo de avaliação, o mais importante passo a se realizar é caracterizar os materiais. A extensão dessa avaliação depende de dados existentes e da natureza e duração do contato do produto com o corpo humano. Caso os processos de fabricação e os constituintes do produto possuam um histórico de uso seguro ao longo dos anos de fabricação, posteriores ensaios podem não ser necessários.

Na condição da decisão pela realização de ensaios clínicos, há alguns critérios a serem seguidos. São eles: (i) o ensaio deve ser realizado no produto final estéril; (ii) a escolha dos procedimentos de ensaio deve considerar a natureza do produto, a atividade toxicológica dos constituintes, a relação da área de superfície do produto que entra em contato com o usuário, a sensibilidade do ensaio e que o objetivo principal do mesmo é garantir a proteção do usuário; (iii) os resultados dos ensaios devem ser reprodutíveis.

Para a posterior realização da avaliação dos ensaios ou do estudo clínico, faz-se necessária a detalhada documentação da estratégia de avaliação, dos critérios de aceitabilidade dos resultados, da adequação dos materiais constituintes, da justificativa para a escolha ou exclusão de ensaios, da interpretação dos dados existentes, da eventual necessidade de dados adicionais e das conclusões sobre a segurança biológica do produto.

Por fim, determina-se que essa norma deva ser utilizada conjuntamente à norma ISO 14971, pois os órgãos regulamentadores solicitam que a avaliação de biocompatibilidade seja realizada dentro de um processo de gerenciamento de risco.

A norma ISO 14971 trata da aplicação de gerenciamento de risco a produtos para a saúde. Tem por objetivo especificar um processo pelo qual um fabricante pode identificar os perigos associados aos produtos para a saúde, incluindo produtos para a 
saúde de diagnóstico in vitro, estimar e avaliar os riscos associados, controlar esses riscos e monitorar a eficácia do controle.

Iniciando o processo, deve-se realizar a análise dos riscos. Para isso, o fabricante deve documentar a finalidade de uso do seu produto e prever possíveis formas errôneas de utilização. Após, deve-se identificar eventuais perigos associados ao uso do produto em condições usuais e também em modo de falha. Além disso, faz-se necessária a suposição de potenciais sequências de eventos adversos que podem ser desencadeadas com base em um único perigo.

Posteriormente, o processo passa para a avaliação dos riscos. A empresa fabricante deve realizar a investigação desses perigos e, com base em critérios anteriormente definidos, decidir pela aceitação, redução, mitigação ou extinção dos riscos.

No caso de a empresa optar por interferir nos perigos, medidas de controle de riscos devem ser tomadas. O fabricante deve priorizar a segurança do projeto do produto, medidas de proteção do processo ou do produto, ou informações de segurança no produto. Essas medidas devem reduzir a probabilidade de ocorrência de danos ou reduzir a severidade dos mesmos.

Após a implementação das medidas de redução, os riscos residuais devem ser avaliados tendo os critérios de aceitabilidade como parâmetro. Se, porventura, estes não sejam aceitos, é preciso que sejam executadas ações adicionais. Subsequentemente, após a aplicação de todas as possíveis providências, o fabricante deve determinar se os riscos residuais gerais do produto são admissíveis.

Por fim, os resultados da análise total dos riscos do produto e seu processo devem ser registrados como um relatório de gerenciamento de risco. Para fins de seguir com um gerenciamento rígido deve-se estabelecer um sistema para coletar e analisar dados de produção e pós-produção ao longo do tempo.

\section{MÉTODO DE PESQUISA}

Nesta seção serão apresentados procedimentos metodológicos necessários para a realização deste trabalho de pesquisa. Foi realizado um breve referencial teórico sobre o tema em estudo. Segundo Miguel (2010), o referencial teórico é importante para que se 
tenha ideia clara do escopo do trabalho, delimitando as fronteiras da investigação, fornecendo suporte teórico e permitindo a observação da evolução do tema em estudo.

De acordo com os objetivos descritos na introdução deste trabalho, utilizou-se uma pesquisa exploratória. Para Mattar et al. (2014), os métodos utilizados são amplos e versáteis, compreendendo: levantamentos em fontes secundárias, levantamentos de experiências, estudos de casos selecionados e observação informal. Para Gil (2017), a pesquisa exploratória é elaborada com o propósito de proporcionar uma visão geral, de caráter aproximativo, a respeito de determinado fato que, ao final do processo, torna-se um problema compreensível, susceptível de investigação mediante uma sistematização de alguns procedimentos.

Com relação à escolha da modalidade de pesquisa, tem-se, neste trabalho, um estudo de caso, que é um monitoramento de projetos e atividades, uma observação da realidade, sem intervenção sistemática do pesquisador (WAZLAWICK, 2014). Em um estudo de caso, os dados são coletados com um propósito específico e os resultados documentados. Segundo Fachin (2017), o estudo de caso pode se caracterizar por um estudo intensivo, em que se leva em conta a compreensão do assunto como um todo, do assunto investigado. Uma das grandes utilidades dos estudos de caso é verificada nas pesquisas exploratórias. Por sua flexibilidade, é recomendável, nas etapas iniciais de uma investigação sobre temas complexos, para a construção de hipóteses ou reformulação do problema (YIN, 2014).

O estudo de caso foi realizado tendo um setor de uma empresa específica como objeto de estudo. $\mathrm{O}$ estudo de caso foi realizado através de três etapas principais, que finalizaram na proposta da sistemática, conforme explicitado na Figura 1.

Primeiramente, fez-se necessário realizar um mapeamento geral do processo escolhido. Isso deve ser realizado para averiguar como se dá o processo produtivo: as entradas e saídas do material, bem como a sequência de produção seguida pelo produto, dentro da linha de produção. No presente trabalho o mapeamento foi realizado utilizando o software Business Process Model and Notation (BPMN). O BPMN é uma notação que foi criada para padronizar a modelagem dos processos das empresas.

Com o mapeamento geral pronto e todos os inputs e outputs determinados, é fundamental que se examine os dados existentes. Através disso é possível verificar se os dados são suficientes para entender os eventuais riscos biológicos. Para a empresa estudada foram compilados dados de quinze anos de reclamações registradas junto à 
empresa e à ANVISA. Juntamente aos responsáveis da empresa ficou definido que seria uma métrica apropriada para analisar quantitativa e qualitativamente o histórico de produção.

Figura 1 Etapas necessárias para a definição da sistemática no estudo de caso

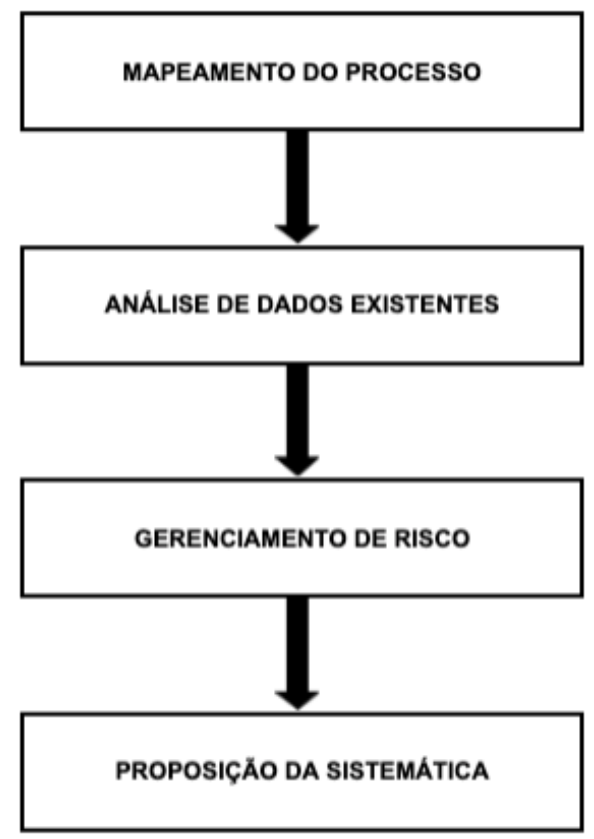

Fonte: Autores (2021)

Assim, passa-se à fase de gerenciamento de risco. Nesta fase deve-se verificar o uso pretendido do material analisado e os potenciais riscos de falha de projeto e processo. Para isso é necessário definir também a duração e o local de contato do produto e como será a utilização do mesmo. O gerenciamento de risco da empresa estudada foi realizado com base na ISO 14971, que serve como guia para tal processo dentro de uma organização que fabrica produtos médicos. Além disso, esta análise foi executada com o aval e acompanhamento da Responsável Técnica pela produção de equipos.

Por fim, com o processo analisado detalhadamente e todos os coletados, chega-se à proposição da sistemática. Ela define os passos que devem ser seguidos para a realização da avaliação biológica final. Essa sistemática foi estabelecida após todo estudo e avaliação do processo e das etapas que foram aplicadas na empresa estudada.

\section{RESULTADO E DISCUSSÕES}

Nessa seção será apresentado o estudo de caso, que foi dividido nas seguintes etapas: (i) Mapeamento do processo; (ii) Análise de dados existentes; (iii) Gerenciamento de risco; (iv) Proposta da sistemática. Ao final da seção apresentada a proposta de como 
deve ser realizada a avaliação sistemática da biocompatibilidade para empresas do ramo médico hospitalar.

\subsection{Mapeamento do Processo}

O processo se inicia com a movimentação da matéria prima. Esta pode ser proveniente de dois setores iniciais: recebimento ou injeção. Esses dois setores enviam então os materiais prontos para o estoque de matéria prima. $\mathrm{O}$ estoque de matéria prima envia então, para o estoque interno do setor de Produção de Equipos, os materiais solicitados que são necessários para a realização das ordens de produção.

O processo produtivo de equipos em si pode ser realizado de duas formas, por esteira ou bancada. A escolha do tipo de produção vai depender do modelo de equipo que deve ser trabalhado. Na esteira, dez colaboradores realizam a montagem do equipo em dez etapas diferentes enquanto na bancada apenas um colaborador realiza toda a montagem do equipo sozinho.

Com a montagem finalizada, os equipos prontos para duas etapas de controle de qualidade. Na primeira etapa, a pesagem, alguns colaboradores são responsáveis por conferir se o peso e a medida do produto estão corretos. E na segunda, o ensaio de estanqueidade, os colaboradores testam o produto para verificar a existência de algum vazamento.

Após os ensaios, os equipos aprovados nos testes passam para a blister, que é a embaladora, onde os equipos são selados dentro de uma embalagem feita de plástico e papel cirúrgico. A seguir, as embalagens passam por uma esteira até o encaixotamento, em que as embalagens são colocadas em caixas fechadas para a comercialização.

As caixas seladas são então enviadas para a esterilização. Nesse estágio, elas são colocadas empilhadas dentro de uma autoclave industrial que é alimentada por óxido de etileno. O óxido é dispersado em sua forma gasosa por algumas horas, e assim é capaz de eliminar qualquer microorganismo patógeno que possa estar presente nos produtos.

Por fim, as caixas esterilizadas passam para o estoque de produto acabado, onde ficam até o momento que devem ser expedidas para atender a algum pedido de venda. $\mathrm{O}$ mapeamento do processo realizado no estudo de caso é apresentado na Figura 6 do Apêndice 1. 


\subsection{Análise dos dados existentes}

Para que as empresas não necessitem realizar testes de biocompatibilidade, um dos pontos exigidos é que exista uma vasta documentação que suporte sua decisão. Assim, necessita-se fazer uso de dados existentes coletados ao longo dos anos de produção.

A empresa estudada conta com um sistema de gerenciamento de reclamações, conforme exigido pelo Ministério da Saúde. Ela estabeleceu, através de uma Política de Tratativa de Reclamações, os requisitos gerais e diretrizes para a vigilância sistematizada de eventos adversos e queixas técnicas de produtos para a saúde na fase póscomercialização, que promovam sua resolução de maneira consistente, sistemática e responsiva, a fim de garantir a proteção e promoção da saúde de seus clientes. Devido a isso, em conformidade com as normas vigentes desse período, a empresa registrou todas as reclamações que foram cadastradas junto à mesma ao longo dos anos de fabricação.

Analisando as reclamações, foi verificado um total de duas mil trezentas e quarenta e seis reclamações registradas ao longo de quinze anos de registro. Dentre as reclamações, catorze se destacam por serem as mais frequentes, representando $29,87 \%$ de todas as reclamações.

Segundo a ISO 10993-1, considerando o tipo de produto, os efeitos biológicos que devem ser considerados para os produtos da empresa são: Citotoxicidade; Sensibilização; Irritação ou reatividade intracutânea; Toxicidade sistêmica (aguda); Hemocompatibilidade. Através da análise de todas as reclamações registradas, a empresa atestou que nenhuma delas tem por motivação os efeitos biológicos que comprovam ou não a biocompatibilidade.

Tendo em vista que não foram registradas reclamações que alegam algum dos efeitos estudados, a análise de dados na empresa estudada se provou infrutífera. Dessa forma, não há dados históricos que possam prover maiores informações de biocompatibilidade dos produtos na empresa em estudo.

\subsection{Gerenciamento de Risco}

Para uma melhor análise geral, nesse estudo de caso, o gerenciamento de risco foi dividido em quatro etapas principais, conforme a Figura 2. A primeira etapa, de 
estabelecimento do contexto, foi trabalhada ao longo da introdução e revisão teórica deste trabalho.

Figura 2 Etapas do Gerenciamento de Risco

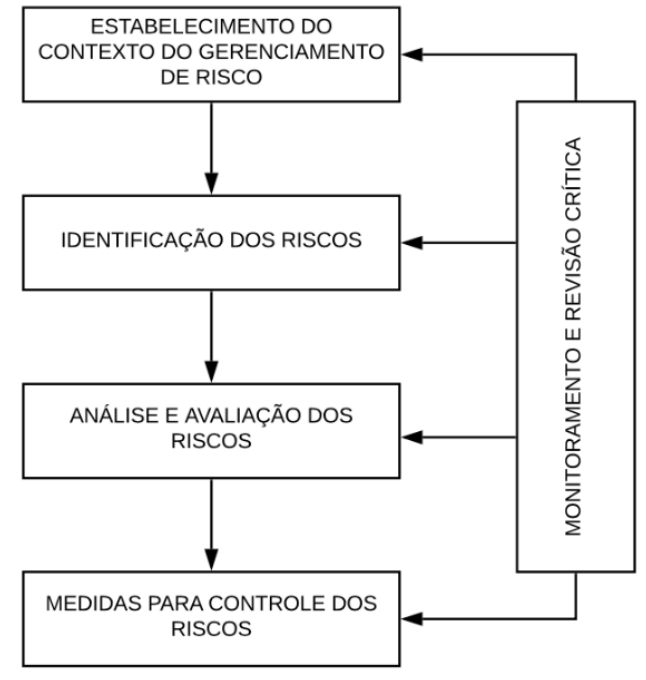

Fonte: Autores (2021)

Para a identificação dos riscos, foi realizada uma análise crítica com base no mapeamento do processo da empresa estudada. Assim, foram identificadas pelo setor do Sistema da Qualidade da empresa juntamente à Direção (representados pela Responsável Técnica e pelo Diretor Operacional da fábrica) três etapas críticas do processo produtivo com relação à biocompatibilidade: R1 o Recebimento, R2 a Injeção e R3 a Esterilização. As etapas críticas podem ser observadas com a marcação em vermelho, conforme apresentado na Figura 6 do Apêndice 1.

Após a identificação das etapas críticas do processo quanto aos riscos de biocompatibilidade, passou-se para as etapas de análise e avaliação dos riscos. Nesse estudo, essas etapas foram realizadas conjuntamente. Assim, nesse estágio os riscos foram compreendidos com base em sua natureza e determinados com base em critérios estabelecidos.

Primeiramente, foram estabelecidos cinco níveis de probabilidade de ocorrência e de consequência dos efeitos biológicos estudados. Esses níveis foram definidos com base na norma ABNT NBR ISO 14971 (2009), que explicita na sua seção D.3.4.1 (Anexo 1) de exemplos de análise qualitativa para o gerenciamento de risco, o seguinte:

Muitas técnicas podem ser utilizadas para a análise qualitativa. Uma maneira típica é usar uma matriz $\mathrm{N}$ por $\mathrm{M}$ para descrever as probabilidades e severidades de risco associadas com cada situação perigosa. Definem-se $\mathrm{N}$ 
níveis de probabilidade e $\mathrm{M}$ níveis de severidade. Cada célula da matriz representa um subconjunto do conjunto global de possíveis riscos. As células são criadas pelo particionamento de uma gama de probabilidades possíveis e uma gama de consequências possíveis' (ABNT NBR ISO 14971 (2009), seção D.3.4.1).

O Quadro 1 apresenta os cinco níveis de probabilidade de ocorrência dos efeitos biológicos.

Quadro 1 - Níveis de probabilidade de ocorrência dos efeitos biológicos

\begin{tabular}{|c|c|}
\hline Probabilidade de ocorrência & Descrição \\
\hline Muito baixo & Raro/Remoto \\
\hline Baixo & Improvável de ocorrer \\
\hline Médio & Pode ocorrer \\
\hline Alto & Provável de ocorrer \\
\hline Muito alto & Frequente/Comum \\
\hline
\end{tabular}

Fonte: Adaptado da norma ISO 14971 (2009)

Posteriormente foram definidos cinco níveis qualitativos consequência. Os níveis qualitativos avaliam a severidade dos danos dos efeitos biológicos em questão, conforme apresentado no Quadro 2.

Quadro 2 - Níveis de severidade dos danos dos efeitos biológicos

\begin{tabular}{|c|c|}
\hline Severidade dos danos & Descrição \\
\hline Desprezível & Não causará lesão \\
\hline Menor & Lesão leve e reversível \\
\hline Moderado & Lesão mais séria, porém, reversível \\
\hline Sério & Lesão irreversível \\
\hline Crítico & Morte \\
\hline
\end{tabular}

Fonte: Adaptado da norma ISO 14971 (2009)

Após a definição dos níveis de probabilidade e severidade, é possível produzir uma matriz de classificação de riscos 5x5. A matriz, apresentada na Figura 3, adaptada de Lapa (2016) para o estudo de caso realizado, classifica os riscos em três faixas diferentes: alto risco (vermelho), médio risco (amarelo) e baixo risco (verde). 
Figura 3 Matriz de classificação dos riscos

\begin{tabular}{|c|c|c|c|c|c|}
\hline \multicolumn{6}{|c|}{ CLASSIFICAÇÃO DOS RISCOS } \\
\hline \multirow{2}{*}{ PROBABILIDADE } & \multicolumn{5}{|c|}{ SEVERIDADE } \\
\hline & Desprezivel & Menor & Moderado & Sério & Crítico \\
\hline Muito baixo & \multirow{2}{*}{\multicolumn{2}{|c|}{ BAIXO RISCO }} & \multirow{3}{*}{ MÉDIO RISCO } & \multirow{4}{*}{\multicolumn{2}{|c|}{ ALTO RISCO }} \\
\hline Baixo & & & & & \\
\hline Médio & & & & & \\
\hline Alto & & & & & \\
\hline Muito alto & & & & & \\
\hline
\end{tabular}

Fonte: Adaptado de Lapa (2016).

Após a finalização da definição dos níveis de risco passamos para a avaliação dos mesmos. Então, após análise crítica, os riscos estimados na fase de identificação foram colocados dentro das células da matriz, conforme apresentado na Figura 4.

Figura 4 Classificação dos riscos identificados no processo estudado

\begin{tabular}{|c|c|c|c|c|c|}
\hline \multicolumn{5}{|c|}{ CLASSIFICAÇÃo DOS RISCOS } \\
\hline \multirow{2}{*}{ PROBABILIDADE } & \multicolumn{4}{|c|}{ SEVERIDADE } \\
\cline { 2 - 6 } & Desprezivel & Menor & Moderado & Sério & Crítico \\
\hline Muito baixo & & & & & \\
\hline Baixo & & & R1 e R2 & & \\
\hline Médio & & & R3 & & \\
\hline Alto & & & & & \\
\hline Muito alto & & & & & \\
\hline
\end{tabular}

Fonte: Adaptado de Lapa (2016).

Os riscos definidos foram classificados na faixa de médio risco, pois tem baixa e média probabilidade de ocorrência e a severidade dos danos que causam é moderada. Por definição da empresa estudada, os níveis de baixo e médio risco são aceitáveis e a faixa de alto risco é inaceitável. Com a classificação finalizada, deu-se início à etapa de controle dos riscos avaliados.

Para a empresa estudada, os riscos avaliados foram de médio impacto. Sendo assim, os responsáveis optaram por aceitá-los. Porém, apesar desse fato, foram apresentadas duas propostas de ação para um melhor controle desse risco. São elas:

1. Criação de formas de testagem química da matéria-prima recebida;

2. Criação/implementação de dispositivos de alarme que não permitam que o produto acabado seja expedido antes do período da quarentena.

Com a aplicação dessas medidas simples, torna-se possível novas avaliações dos riscos do processo produtivo da empresa estudada. Tendo assim, grandes chances de ocorrer a diminuição da probabilidade de ocorrências de risco. Consequentemente, esse fato levaria os riscos à uma melhora na sua classificação, passando de médio risco para baixo risco. 


\subsection{Proposta de Sistemática para avaliação da Biocompatibilidade}

A partir da realização do estudo de caso foi possível a proposição de uma sistemática para a avaliação da biocompatibilidade de produtos médico-hospitalares. Os passos da proposta de sistemática para a avaliação da biocompatibilidade são apresentados na Figura 5.

Figura 5 Passos da proposta de Sistemática para avaliação de biocompatibilidade

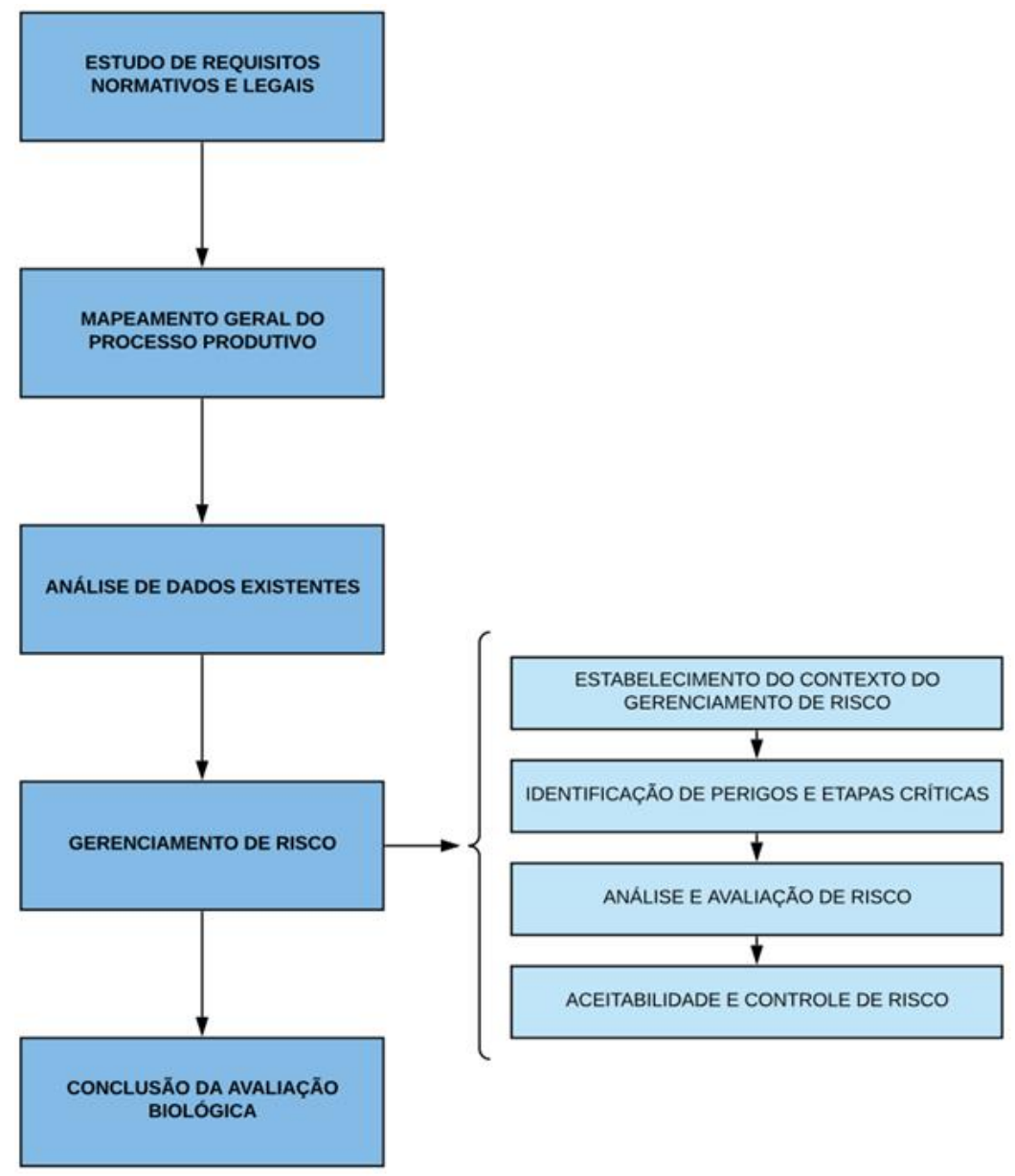

Fonte: Autores (2021)

Primeiramente, o processo para a avaliação de biocompatibilidade deve ser iniciado com o estudo do avaliador acerca das normas ISO 10993 (Avaliação biológica de produtos para a saúde) e ISO 14971 (Produtos para a saúde - Aplicação de gerenciamento de risco a produtos para a saúde). Na sequência, deve ser realizado o mapeamento geral do processo produtivo da empresa estudada. É através do mapeamento que o gerenciamento de riscos será realizado. 
Posteriormente, passa-se à análise de dados existentes. Nessa fase, serão beneficiadas empresas que possuem documentação e registros extensos de produção. Logo após, inicia-se a fase de gerenciamento de risco. Aqui, existem diferentes escolhas para o avaliador optar em como desenvolver a gestão de riscos. Como apresentado neste trabalho, a sugestão é de que seja seguida a série lógica apresentada na Figura 6, contendo os seguintes passos: (i) Estabelecer o contexto do gerenciamento de risco; (ii) Identificar os riscos e perigos; (iii) Analisar e avaliar os riscos - aqui entende-se que as duas etapas são simultâneas e inerentes uma à outra; (iv) Definir a aceitabilidade do risco e medidas de controle para o mesmo.

Com a finalização de todos esses estágios, a empresa que está realizando a avaliação biológica pode então finalizar a verificação emitindo um parecer que atesta ou não a biocompatibilidade de seus produtos.

\section{CONSIDERAÇÕES FINAIS}

Tendo em vista o uso pretendido dos produtos médicos e hospitalares, a avaliação biológica é fundamental para que o fabricante possa garantir a segurança de seu produto e também assegurar sua confiabilidade dentro do mercado. Assim, atesta-se seu comprometimento como produtor do setor em questão para com os pacientes e usuários.

Isso posto, o presente trabalho buscou propor uma sistemática para a avaliação da biocompatibilidade dos produtos de uma empresa do ramo médico-hospitalar. Tinha por objetivos estudar as normas de biocompatibilidade existentes, apresentar dados de como uma avaliação de biocompatibilidade deve ser realizada, definir critérios a serem seguidos para a implementação da avaliação e servir de guia para futuros trabalhos no Brasil.

O trabalho foi desenvolvido através de uma metodologia apoiada em cinco principais etapas. Inicialmente foi realizada uma revisão de literatura, com o intuito de basear o trabalho teoricamente, estudando-se sobre temas como desenvolvimento de produtos, mapeamento de processos e segurança biológica. Passou-se então para o estudo de normas e requisitos normativos. Essa pesquisa se fundamentou em duas normas, ISO 10993 e ISO 14971, as quais foram extensamente examinadas e aprofundadas. Posteriormente foram analisados casos já existentes em literatura, para se verificar os 
procedimentos metodológicos e avaliativos de tais estudos. O próximo passo foi a realização de um estudo de caso.

O estudo de caso se iniciou com o mapeamento do processo produtivo da empresa, que identificou toda a sequência de processamento dos equipos. Nessa etapa foram realizados todos os passos necessários para que se pudesse propor e comprovar a sistemática planejada. Em seguida foram analisados os dados e documentação existente nos registros da empresa. Aqui, apesar de dados interessantes para uma avaliação de qualidade de produto terem sido compilados, eles não foram relevantes para este estudo. Assim, passou-se para a etapa de gerenciamento de risco. Nessa fase foram identificados, analisados e avaliados os riscos do processo com relação aos efeitos biológicos considerados na avaliação biológica. Por fim, foram propostas duas recomendações para a diminuição dos riscos do processo.

Com isso, o estudo de caso foi finalizado com a proposição de uma sistemática de avalição de biocompatibilidade. A mesma foi proposta com uma divisão de cinco etapas elementares, sendo elas: (i) estudo de requisitos normativos e legais; (ii) mapeamento do processo; (iii) análise de dados existentes; (iv) gerenciamento de risco; (v) parecer conclusivo da avaliação biológica. Essas etapas são genéricas e aplicáveis a qualquer empresa do ramo de produtos e artigos médicos e hospitalares.

Conclui-se que o presente trabalho cumpriu seus objetivos iniciais. Além de auxiliar a empresa estudada a realizar sua própria avaliação de biocompatibilidade, gerou um método para que outras empresas possam replicar o estudo futuramente. Como sugestão para trabalhos futuros, sugere-se a continuação do gerenciamento de risco com a aplicação das medidas de controle propostas: criação de formas de testagem química da matéria-prima recebida e criação/implementação de dispositivos de alarme que não permitam que o produto acabado seja expedido antes do período da quarentena. Além disso, é pertinente a realização da verificação posterior à aplicação das medidas. Assim, efetiva-se o ciclo total do gerenciamento de risco.

\section{REFERÊNCIAS}

Clark, K. B.; Fujimoto, T. (1991) Product development performance: strategy, organization and management in the world auto industry. 1. ed. Boston, Massachusetts: Harvard Business School Press. 
Cooper, R. G. (2018) Perspective: the stage-gate® idea-to-launch process - update, What's New, and NexGen Systems. Journal of Product Innovation Management, v. 25, p. 213-232.

Fachin, O. (2017) Fundamentos de metodologia. $6^{\text {a }}$ ed. São Paulo: Saraiva.

Ferreira, A. B. H. (2014) Dicionário Aurélio. 5. ed. São Paulo: Editora Positivo.

Fleury, A.; Mello, C. H. P; Nakano, D. N.; Lima, E. P. D.; Turrioni, J. B.; Lee Ho, L.; Morabito, R.; Costa, S. E. G. D.; Martins, R. A.; Souza, R.; Pureza, V. (2018)

Metodologia de Pesquisa em Engenharia de Produção e Gestão de Operações, $3^{\mathrm{a}}$ ed. Coordenação: CAUCHICK-MIGUEL, P. A. Rio de Janeiro: Elsevier.

Gil, A. C. (2017) Como Elaborar Projetos de Pesquisa. 6a ed. São Paulo: Atlas.

Lapa, R. P. (2016) Valoração do Risco. Disponível em:

$<$ http://segurancatemfuturo.com.br/index.php/home/gerenciamento-de-riscos/valoracaodo-risco/>. Acesso em: 21 de Agosto de 2020.

Marshall Junior, I.; Rocha, A. V.; Mota, E. B.; Quintella, O. M. (2012) Gestão da Qualidade e Processos. 1. ed. Rio de Janeiro: Editora FGV.

Mattar, F. N.; Oliveira, B.; Motta, S. (2014) Pesquisa de Marketing: metodologia, planejamento, execução e análise. 7a ed. Rio de Janeiro: Elsevier.

Rozenfeld, H.; Forcellini, F.; Amaral, D. C.; Toledo, J.; Alliprandini, D.; Scalice, R. (2006) Desenvolvimento de Produto: Uma referência para Melhoria do Processo. 1. ed. São Paulo, Editora Saraiva.

Tinkler, J. J. B. (2001) Biological Safety \& European Medical Device Regulations. 1. ed. London: Quality First International Press.

Toledo, J. C.; Silva, S. L.; Mendes, H. S.; Jugend, D. (2008) Fatores críticos de sucesso no gerenciamento de projetos de desenvolvimento de produto em empresas de base tecnológica de pequeno e médio porte. Gestão \& Produção, v. 15, p. 117-134.

Wazlawick, R. S. (2014) Metodologia de Pesquisa para Ciência da Computação. Rio de Janeiro: Elsevier.

Wheelwright, S. C.; Clark, K.B. (1992) Revolutionizing new product development: quantum leaps in speed, efficiency and quality. 1. ed. New York: The Free Press.

Yin, R. K. (2014) Estudo de Caso: planejamento e métodos. $5^{\mathrm{a}}$ ed. Porto Alegre: Bookman. 


\section{Apêndice 1}

Figura 6 - Mapeamento do Processo em estudo, em vermelho etapas críticas

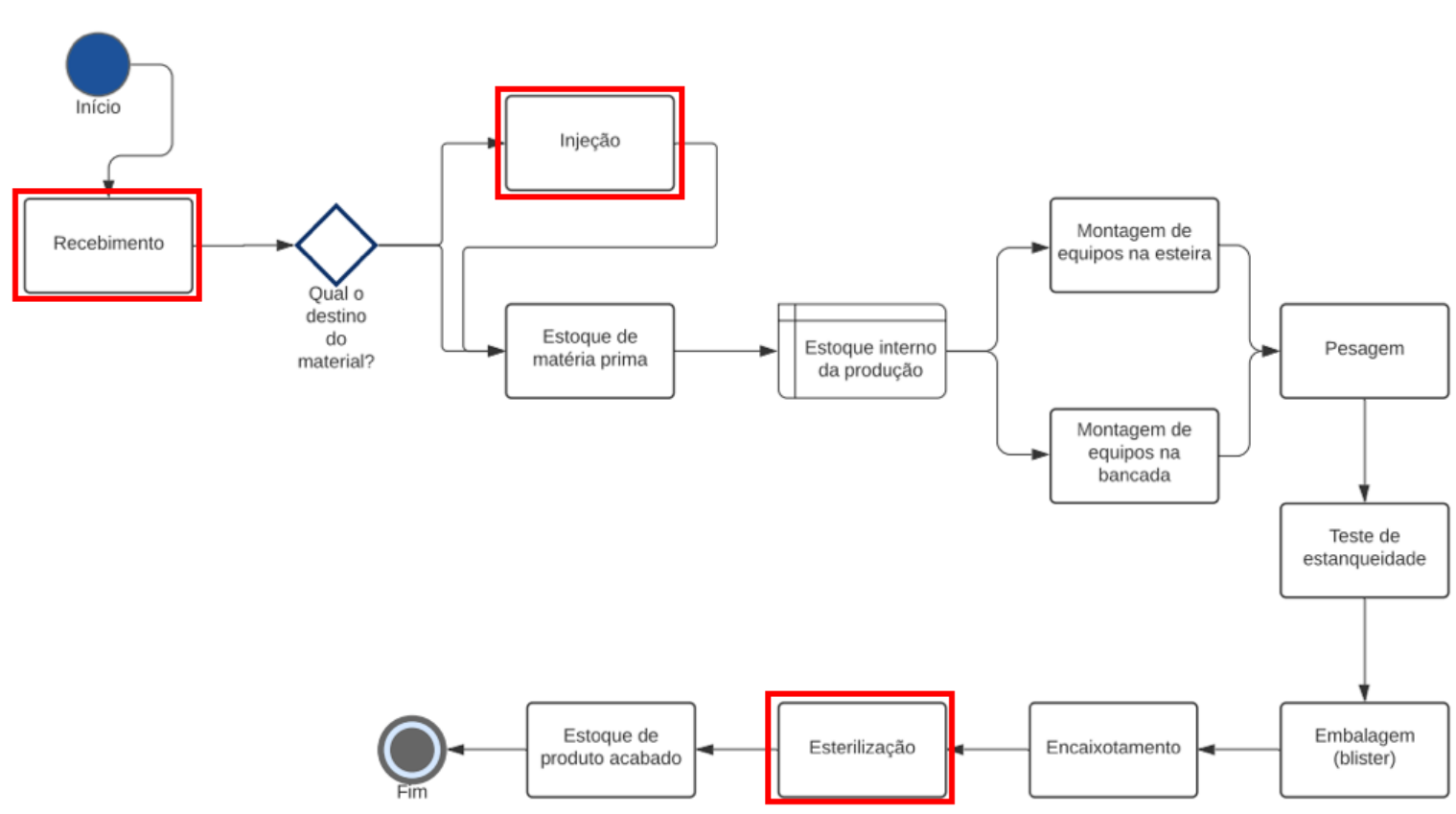

Fonte: Autores (2021) 


\section{Anexo 1}

Parte da norma ABNT NBR ISO 14971 (2009), que explicita na sua seção D.3.4.1 (Anexo 1) - de exemplos de análise qualitativa para o gerenciamento de risco.

ABNT NBR ISO 14971:2009

\section{D.3.4 Exemplos}

D.3.4.1 Análises qualitativas

Muitas técnicas podem ser utilizadas para a análise qualitativa. Uma maneira tipica é usar uma matriz $\mathrm{N}$ por $\mathrm{M}$ para descrever as probabilidades e severidades de risco associadas com cada situaçăo perigosa. (a) un sunto do conlilades possiveis $3 \times 3$ baseada nas definicões encontradas nas Tabelas D.1 e D.2. Recomenda-se aos fabricantes tornar essas definiçōes tåo especificas ao produto e explicitas quanto necessário para assegurar sua utilizaçăo de forma reprodutivel.

Tabela D.1 - Exemplos de niveis qualitativos de severidade

\begin{tabular}{|c|c|}
\hline Termos comuns & Descriçăo possivel \\
\hline Significativo & Morte ou perda de funçăo ou estrutura \\
\hline Moderado & Lesăo reversível ou de importância menor \\
\hline Desprezivel & Não causará lesăo ou irá lesionar levemente \\
\hline
\end{tabular}

Tabela D.2 - Exemplos simplificados de

\begin{tabular}{|c|c|}
\hline Termos comuns & Descriçăo possivel \\
\hline Alto & Provável de ocorrer, freqüente, comum \\
\hline Médio & Pode ocorrer, mas năo frequentemente \\
\hline Baixo & Improvável de correr, raro, remoto \\
\hline
\end{tabular}

Usando a probabilidade como linhas e a severidade de dano como colunas, é produzida uma matriz $3 \times 3$. Os riscos estimados ( $R 1, R 2, R 3, \ldots)$ săo posicionados dentro das células apropriadas. O resultado é mostrado na Figura D.2.

Niveis de severidade qualitativa

\begin{tabular}{|c|c|c|c|c|}
\multirow{4}{*}{$\begin{array}{c}\text { Niveis qualitativos de } \\
\text { probabilidade }\end{array}$} & & Desprezivel & Moderado & Significativo \\
\cline { 2 - 5 } & Alto & R1 & R2 & \\
\cline { 2 - 5 } & Médio & & R4 & R5, R6 \\
\hline & Baixo & & R3 & \\
\cline { 2 - 5 } & & & &
\end{tabular}

Figura D.2 - Exemplo de uma matriz de risco qualitativa $3 \times 3$ 


\section{AUTORES :}

\section{Ariane Ferreira Porto Rosa}

Doutora em Automatique et Informatique Appliquées (Spécialité Génie Industriel Qualité), pela Université de Nantes.

Instituição: Universidade Federal de Pelotas, Centro de Engenharias, Curso de Engenharia de Produção.

Endereço: Rua Benjamin Constant, 989, Bairro Porto, Pelotas-RS, Brasil.

E-mail: afprosa61@gmail.com

Lattes: lattes.cnpq.br/7662929784136459

Researchgate: www.researchgate.net/profile/Ariane_Ferreira_Porto_Rosa

Orcid: https://orcid.org/0000-0002-1345-7523

\section{Geórgia Souto}

Bacharel em Engenharia de Produção UFPel

Instituição: Centro de Engenharias, Universidade Federal de Pelotas

Endereço: Centro de Engenharias, Prédio da Antiga Cotada, Rua Benjamin Constant, 989 - Porto, Pelotas - RS, Brasil - 96010-020

E-mail: soutogeorgia0@gmail.com

\section{Rogério Royer}

Doutor em Administração de Empresas pela Universidade Federal do Rio Grande do Sul.

Instituição: Universidade Federal de Pelotas, Centro de Engenharias, Curso de Engenharia de Produção.

Endereço: Rua Benjamin Constant, 989, Bairro Porto, Pelotas-RS, Brasil.

E-mail: rogroyer@gmail.com

Lattes: http://lattes.cnpq.br/3970416599840926

ORCID: https://orcid.org/0000-0002-1535-1562

\section{Renata Heidtmann BEMVENUTI}

Doutora em Engenharia e Ciência de Alimentos pela Universidade Federal do Rio Grande.

Instituição: Universidade Federal de Pelotas, Centro de Engenharias, Curso de Engenharia de Produção.

Endereço: Rua Benjamin Constant, 989, Bairro Porto, Pelotas-RS, Brasil.

E-mail: renatabemvenuti@gmail.com

Lattes: http://lattes.cnpq.br/3801560326998532 